\title{
Vibro-Impact System Based on Forced Oscillations of Heavy Mass Particle along a Rough Parabolic Line
}

\author{
Srdjan Jović, Vladimir Raičević, and Ljubiša Garić \\ Faculty of Technical Sciences, University of Priština, Knez Miloš Street, No. 7, \\ 38220 Kosovska Mitrovica, Serbia \\ Correspondence should be addressed to Srdjan Jović, jovic003@gmail.com
}

Received 22 January 2012; Accepted 30 March 2012

Academic Editor: Mohammad Younis

Copyright (C 2012 Srdjan Jović et al. This is an open access article distributed under the Creative Commons Attribution License, which permits unrestricted use, distribution, and reproduction in any medium, provided the original work is properly cited.

\begin{abstract}
This paper analyses motion trajectory of vibro-impact system based on the oscillator moving along the rough parabolic line in the vertical plane, under the action of external single-frequency force. Nonideality of the bond originates of sliding Coulomb's type friction force with coefficient $\mu=\operatorname{tg} \alpha_{0}$. The oscillator consists of one heavy mass particle whose forced motion is limited by two angular elongation fixed limiters. The differential equation of motion of the analyzed vibro-impact system, which belongs to the group of common second order nonhomogenous nonlinear differential equations, cannot be solved explicitly (in closed form). For its approximate solving, the software package WOLFRAM Mathematica 7 is used. The results are tested by using the software package MATLAB R2008a. The combination of analytical-numerical results for the defined parameters of analyzed vibro-impact system is a base for the motion analysis visualization, which was the primary objective of this analytic research. Upon the phase portrait of the heavy mass particle obtained, the energy of the considered vibro-impact system is analyzed. During the graphical visualization of the energetic changes, one of the steps is the process of the phase trajectory equations determination. For this determination, we have used interpolation process that utilizes Lagrange interpolation polynomial.
\end{abstract}

\section{Introduction}

Research on the vibro-impact systems impact and the dynamics of nonlinear phenomena at presence of the certain discontinuities represents an area of interest for the researches all over the world. The theoretical knowledge on vibro-impact systems (see reference [1-5]) is of particular importance for engineering practice, due to wide occurrence of vibro-impact actions used in technological processes realization. Based on the modern knowledge on vibroimpact systems theory, and considering the papers with this theme of the following authors: 
Peterka [6-8], Hedrih [9-12], Nayfeh and his associates [13, 14], Dimentberg, and Menyailov [15], Foole and Bishop [16], Luo and Xie [17], Nordmark [18], Pavlovskaia, and Wiercigroch $[19,20]$ et al., we can say that today we have raised interest for investigation of energy transfers inside the complex systems and nonlinear modes. Because of that, it is important to study energetic analysis of vibro-impact processes dynamics in the vibro-impact systems with one or more degrees of freedom.

Needed theoretical knowledge that introduces us in the problems of this paper is obtained from the works of Hedrih $[10,11]$ which are related to the moving of the heavy mass particle along the rough curved trajectories. In these papers, basic mathematical description of a heavy mass particle trajectory along rough curved lines is given, and as a special case, motions of the heavy mass particle along the rough circular line, rough cycloid curve, and rough parabola line are given.

Relying on the previous works of the coauthor of this paper [21-26], in which were analyzed more various vibro-impact systems, with one and two degrees of freedom, on the basis of free and forced oscillations of heavy mass particle that is moving along rough curved lines such as parabola, circle and cycloid, Coulomb's sliding friction and with limited elongations, in this paper, a vibro-impact system with one degree of freedom on the basis of forced oscillations of the heavy mass particle, with mass $m$, moving along a rough parabolic line in the vertical plane, coefficient of sliding friction with limited angular elongations is studied (Figure 1). On the heavy mass particle acts the external single-frequency (periodical) force, $F(t)=F_{0} \cos \Omega t$, where $F_{0}$ is corresponding forces amplitude and $\Omega$ is frequency of the external force. Positions of the elongation limiters are determined by angular coordinates $s_{\mathrm{ul}, 1}=s_{1}\left(\varphi_{1}\right)$ and $s_{\mathrm{ul}, 2}=s_{2}\left(\varphi_{2}\right)$ which are referenced to the equilibrium position of a particle. Arc (curved) coordinates are given as functions of angle $\varphi$. This angle is an angle between tangent and $O x$ axis. Arc coordinate $s(\varphi)$ is an independent (generalized) coordinate of the system, providing the system has one degree of freedom. At the start moment, heavy mass particle was on the $s_{0}\left(\varphi_{0}\right)$ distance from the equilibrium position, and received initial velocity $v_{0}=\dot{s}_{0}$.

Our task is to consider properties of forced oscillations of a heavy mass particle moving along the rough parabolic line with limited elongation, wherefore the system becomes vibroimpact type with two limiters, and two-sided limited elongation. For each motion interval, the forced motion differential equations are needed. Also, the conditions of friction forces alternations, elongation limiting of the system, and motion initialization are added to the differential equations. Alteration of the friction force direction is related to the alteration of the heavy mass particle angular velocity direction. Also, phase trajectories equation (using interpolation procedure, by means of Lagrange interpolation polynom) in the phase plane $(\varphi, \dot{\varphi})$ and constant energy curves equations should be determined. Corresponding graphic visualization and representative point of the system kinetic state motion during the kinetics (dynamics) should be worked out, too. Similarly, deviation of the total system mechanical energy during the motion and rate of the mechanical energy decrease in each characteristic motion interval should be analyzed. Determine also the number of impacts, at which the system does not behave as a vibro-impact one.

\section{Differential Equation of a Heavy Mass Particle Oscillation along a Rough Parabolic Line}

Using the principle of dynamic equilibrium (see references $[5,11]$ ), we can write equation of a heavy mass particle motion along the curvilinear path in the following form: $\vec{I}_{F}+\vec{G}+\vec{F}_{N}+\vec{F}_{\mu}=$ 0 . 


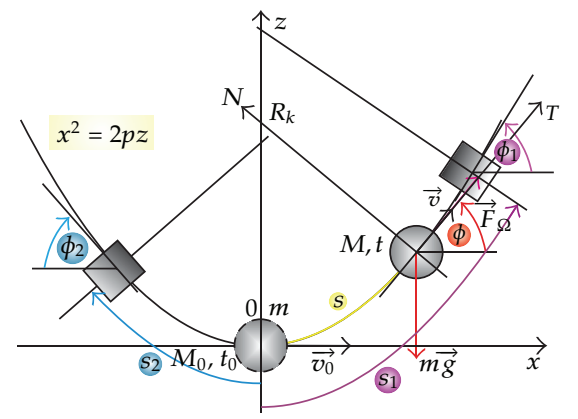

(a)

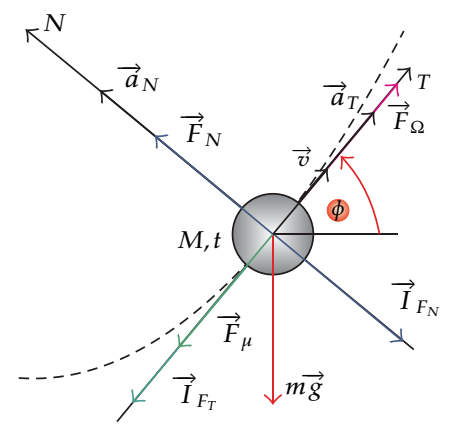

(b)

Figure 1: Two fixed elongation limiter systems, on the basis of the oscillator with one heavy mass particle, external single-frequency force driven. (a) Starting and dislocated position of a heavy mass particle; (b) force plan.

The inertial force originating of heavy mass particle acceleration $\left(\vec{a}=\vec{a}_{N}+\vec{a}_{T}\right)$ has two components, one in tangential and second one in perpendicular direction, so we can write

$$
\vec{I}_{F}=-m \vec{a}_{N}-m \vec{a}_{T}=-m \frac{v^{2}}{R_{k}} \vec{N}-m \dot{v} \vec{T}
$$

where $R_{k}$ is a path curve radius in the instant position point of the heavy mass particle. $\vec{N}$ and $\vec{T}$ are the unit vectors (orts) of the inertial force main orthogonal and tangential components at instant position point on the rough curvilinear line.

Using the curvilinear natural coordinate system, previous vector equation (2.1) can be written in the following form:

$$
(-m \ddot{s} \vec{T})+\left(-m \frac{v^{2}}{R_{k}} \vec{N}\right)+m g(-\sin \alpha \vec{T}-\cos \alpha \vec{N})+F_{N} \vec{N}-\mu\left|\vec{F}_{N}\right| \frac{\vec{v}}{|\vec{v}|}=0
$$

where $\alpha=\operatorname{arctg} z^{\prime}$ is angle between tangent and $O x$ axis; and $s$ is the curvilinear coordinate that denotes position of the heavy mass particle on the rough curvilinear path $(d s=$ $\left.d x \sqrt{1+z^{\prime 2}}\right)$. 
After the scalar multiplying of (2.2) with orts $\vec{T}$ and $\vec{N}$, we obtain two scalar differential equations

$$
-m \frac{v^{2}}{R_{k}}-m g \cos \alpha+F_{N}=0 ; \quad-m \ddot{s}-m g \sin \alpha \mp \mu F_{N}=0 .
$$

From the second equation of equation system (2.3) we can obtain perpendicular component intensity of reaction bond in the following shape: $F_{N}=m\left(v^{2} / R_{k}\right)+m g \cos \alpha$. Substituting of this equation in the first equation of the system (2.3) we obtain

$$
\ddot{s}+g \sin \alpha \pm \mu\left(\frac{v^{2}}{R_{k}}+g \cos \alpha\right)=0 .
$$

Assuming that equation of arbitrary curvilinear line in the plane is defined as $z=f(x)$, it follows

$$
\begin{gathered}
\dot{v}=\frac{d v}{d t}=\frac{d}{d t} \dot{s}=\frac{d^{2} s}{d t^{2}}=\ddot{s}=\frac{d}{d t}\left(\dot{x} \sqrt{1+z^{\prime 2}}\right) ; \quad R_{k}=\frac{\sqrt{\left(1+z^{\prime 2}\right)^{3}}}{z^{\prime \prime}} ; \quad \frac{v^{2}}{R_{k}}=\dot{x}^{2} \frac{z^{\prime \prime}}{\sqrt{1+z^{\prime 2}}} ; \quad \operatorname{tg} \alpha=z^{\prime} ; \\
\sin \alpha=\frac{\operatorname{tg} \alpha}{\sqrt{1+\operatorname{tg}^{2} \alpha}}=\frac{z^{\prime}}{\sqrt{1+z^{\prime 2}}}=\frac{d z}{d s}, \quad \cos \alpha=\frac{1}{\sqrt{1+\operatorname{tg}^{2} \alpha}}=\frac{1}{\sqrt{1+z^{\prime 2}}}=\frac{d x}{d s} .
\end{gathered}
$$

If expressions given with (2.5) are embedded in differential equation of a heavy mass particle trajectory along arbitrary curvilinear rough line with slide friction coefficient $\mu$ (2.4), we obtain

$$
\frac{d}{d t}\left(\dot{x} \sqrt{1+z^{\prime 2}}\right)+\frac{1}{\sqrt{1+z^{\prime 2}}}\left[g z^{\prime} \pm \mu\left(\dot{x}^{2} z^{\prime \prime}+g\right)\right]=0
$$

The analysis of this motion represents special case of the general case conducted for motion of a heavy mass particle along the curvilinear rough line.

General parabola equation is $x^{2}=2 p z$, where $2 p[m]$ is the parabola parameter corresponding to the quadruple value of parabola focus-vertex distance.

Using the differential equations (2.4), (2.5), and corresponding expressions in the given system for $z=x^{2} / 2 p, z^{\prime}=d z / d x=x / p$, and $z^{\prime \prime}=1 / p$, we obtain differential equations for motion of a heavy mass particle along the rough parabolic line

$$
\frac{d}{d t}\left(\dot{x} \frac{1}{p} \sqrt{p^{2}+x^{2}}\right)+\frac{g x}{\sqrt{p^{2}+x^{2}}} \pm \frac{\mu}{\sqrt{p^{2}+x^{2}}}\left(\dot{x}^{2}+g p\right)=0
$$


During the solving process of the differential equation (2.7) we introduce a new variable in the form $u=\dot{x}^{2}$. Differencing the new variable expression, we get relations $d u / d x=$ $d\left(\dot{x}^{2}\right) / d x=2 \dot{x}(d \dot{x} / d x)$ and $\ddot{x}=(1 / 2)(d u / d x)$. Considering all given relations, differential equation for motion of a heavy mass particle along the rough parabolic line can be written in the following form:

$$
\frac{d u}{d x}+2 u \frac{1}{p^{2}+x^{2}}(x \pm \mu p)=-\frac{2 g p}{p^{2}+x^{2}}(x \pm \mu p) .
$$

We have transformed nonlinear differential equation describing a heavy mass particle motion along the rough parabolic line (2.7) by introducing a new generalized coordinate $u$, into the common linear first order differential equation with variable coefficients (2.8) of this form $d u / d x+P(x) u=Q(x)$. The solution of this differential equation is $u(x)=$ $e^{-\int P(x) d x}\left[\int Q(x) e^{\int P(x) d x} d x+C\right]$, and for the given case, coefficients of differential equation are

$$
P(x)=\frac{2}{p^{2}+x^{2}}(x \pm \mu p), \quad \begin{aligned}
& z a v>0, \\
& z a v<0,
\end{aligned} \quad Q(x)=-\frac{2 g p}{p^{2}+x^{2}}(x \pm \mu p), \quad \begin{aligned}
& \text { for } v>0, \\
& \text { for } v<0 .
\end{aligned}
$$

The first general integral of differential equation (2.10) has this form

$$
u(x)=\dot{x}^{2}=e^{-\int\left(2 /\left(p^{2}+x^{2}\right)\right)(x \pm \mu p) d x}\left[\int-\frac{2 g p}{p^{2}+x^{2}}(x \pm \mu p) e^{\int\left(2 /\left(p^{2}+x^{2}\right)\right)(x \pm \mu p) d x} d x+C\right] .
$$

Solving the differential equation (2.10) we obtain an equation for the phase trajectory in the phase plane $(x, \dot{x})$

$$
\dot{x}^{2}=e^{-\left[\ln \left(p^{2}+x^{2}\right) \mp 2 \mu \operatorname{arctg}(p / x)\right]}\left[g p\left(p^{2}+x^{2}\right)\left(-e^{\mp 2 \mu \operatorname{arctg}(p / x)}\right)+C\right]
$$

or

$$
\dot{x}^{2}=\frac{1}{\left(p^{2}+x^{2}\right)} e^{ \pm 2 \mu \operatorname{arctg}(p / x)}\left[g p\left(p^{2}+x^{2}\right)\left(-e^{\mp 2 \mu \operatorname{arctg}(p / x)}\right)+C\right] .
$$

Furthermore, we will show the first general integral of differential equation (2.4) in function of the generalized coordinate $\varphi$.

The differential of the arc (curvilinear) path in function of generalized coordinate $\varphi$ is

$$
\begin{gathered}
d s=\sqrt{(d z)^{2}+(d x)^{2}}=\frac{p}{\cos ^{2} \varphi} \sqrt{1+\operatorname{tg}^{2} \varphi} d \varphi, \\
\text { gde je: } \frac{d z}{d x}=\operatorname{tg} \varphi, \quad d x=\frac{p}{\cos ^{2} \varphi} d \varphi, \quad d z=\frac{p \operatorname{tg} \varphi}{\cos ^{2} \varphi} d \varphi .
\end{gathered}
$$


The heavy mass particle moving along the rough parabolic line speed can be calculated with the following formula:

$$
\dot{s}=\frac{p}{\cos ^{2} \varphi} \sqrt{1+\operatorname{tg}^{2} \varphi} \dot{\varphi}=\frac{p}{\cos ^{3} \varphi} \dot{\varphi}
$$

For the given case, expressions (2.5) look like this

$$
\begin{gathered}
\sin \varphi=\frac{z^{\prime}}{\sqrt{1+z^{\prime 2}}}=\frac{x}{\sqrt{p^{2}+x^{2}}}, \quad \cos \varphi=\frac{p}{\sqrt{p^{2}+x^{2}}}, \quad \operatorname{tg} \varphi=\frac{x}{p} \\
R_{k}=\frac{\sqrt{\left(1+z^{\prime 2}\right)^{3}}}{z^{\prime \prime}}=p \sqrt{\left(\frac{p^{2}+x^{2}}{p^{2}}\right)^{3}}=p \sqrt{\left(\frac{\sqrt{p^{2}+x^{2}}}{p} \frac{\sqrt{p^{2}+x^{2}}}{p}\right)}=p \sqrt{\left(\frac{1}{\cos \varphi} \frac{1}{\cos \varphi}\right)^{3}}=\frac{p}{\cos ^{3} \varphi} ; \\
\frac{v^{2}}{R_{k}}=\frac{\dot{s}^{2}}{R_{k}}=\frac{p}{\cos \varphi}\left(1+\operatorname{tg}^{2} \varphi\right) \dot{\varphi}^{2}=\frac{p}{\cos ^{3} \varphi} \dot{\varphi}^{2} .
\end{gathered}
$$

By introducing the previous expressions and expression for

$$
\ddot{s}=\frac{p}{\cos ^{2} \varphi} \sqrt{1+\operatorname{tg}^{2} \varphi} \ddot{\varphi}+\frac{p}{\cos ^{2} \varphi} \operatorname{tg} \varphi \sqrt{1+\operatorname{tg}^{2} \varphi}\left(2+\frac{1}{\cos ^{2} \varphi} \frac{1}{1+\operatorname{tg}^{2} \varphi}\right) \dot{\varphi}^{2}=\frac{p}{\cos ^{3} \varphi} \ddot{\varphi}+\frac{3 p \sin \varphi}{\cos ^{4} \varphi} \dot{\varphi}^{2},
$$

differential equation for the motion of a heavy mass particle along the rough parabolic line, in the function of the generalized coordinate $\varphi$, gets this form

$$
\ddot{\varphi}+(3 \operatorname{tg} \varphi \pm \mu) \dot{\varphi}^{2}+\frac{g \cos ^{3} \varphi}{p}(\sin \varphi \pm \mu \cos \varphi)=0, \quad \begin{array}{ll}
\text { for } v>0 \\
\text { for } v<0 .
\end{array}
$$

By linearization of differential equation (2.17) (introducing the new variable $u=\dot{\varphi}^{2}$ ) we get this form

$$
\frac{d u}{d \varphi}+2 u(3 \operatorname{tg} \varphi \pm \mu)=-\frac{2 g \cos ^{3} \varphi}{p}(\sin \varphi \pm \mu \cos \varphi)
$$

We are looking for a solution of this differential equation (2.18) in the shape $u(\varphi)=$ $e^{-\int P(\varphi) d \varphi}\left[\int Q(\varphi) e^{\int P(\varphi) d \varphi} d \varphi+C\right]$, where (for the given case) differential equation coefficients are as follows:

$$
P(\varphi)=2(3 \operatorname{tg} \varphi \pm \mu), \quad \begin{array}{ll}
\text { for } v>0, & Q(\varphi)=-\frac{2 g \cos ^{3} \varphi}{p}(\sin \varphi \pm \mu \cos \varphi),
\end{array} \quad \begin{aligned}
& \text { for } v>0 \\
& \text { for } v<0,
\end{aligned}
$$


The first general integral of a linear differential equation (2.18) gets this shape

$$
u(\varphi)=\dot{\varphi}^{2}=e^{-\int 2(3 \operatorname{tg} \varphi \pm \mu) d \varphi}\left[\int-\frac{2 g \cos ^{3} \varphi}{p}(\sin \varphi \pm \mu \cos \varphi) e^{\int 2(3 \operatorname{tg} \varphi \pm \mu) d \varphi} d \varphi+C\right] .
$$

Arranging (2.20) we obtain phase trajectory equation, in the phase plane $(\varphi, \dot{\varphi})$, in the form

$$
\dot{\varphi}^{2}=\cos ^{6} \varphi\left(-\frac{g}{p \cos ^{2} \varphi}+C e^{\mp 2 \mu \varphi}\right), \quad \begin{aligned}
& \text { for } v>0, \\
& \text { for } v<0
\end{aligned}
$$

where $C$ is integration constant that depends on starting motion conditions. Depending on motion period of a heavy mass particle along the rough parabolic curve, the integration constant is changing. This period is limited on the phase trajectory with zero velocity points. This change is related to the change of a heavy mass particle motion direction, namely, the velocity direction change that causes also the change of a friction force direction.

If we introduce an effect of the external single-frequency force in our system, differential equation for forced motion of a heavy mass particle along the parabolic line is

$$
\ddot{\varphi}+(3 \operatorname{tg} \varphi \pm \mu) \dot{\varphi}^{2}+\frac{g \cos ^{3} \varphi}{p}(\sin \varphi \pm \mu \cos \varphi)=\frac{F_{0} \cos ^{3} \varphi}{p} \cos \Omega t, \quad \begin{array}{ll}
\text { for } v>0 \\
\text { for } v<0 .
\end{array}
$$

For a complete description of a heavy mass particle dynamics, it is necessary to join the following conditions to the motion differential equation.

$a^{*}$ initial conditions

$$
\begin{gathered}
s_{(0)}\left(\varphi_{(0)}\right)=s_{0}\left(\varphi_{0}\right), \\
v_{(0)}\left(\varphi_{(0)}, \dot{\varphi}_{(0)}\right)=\dot{s}_{(0)}\left(\varphi_{(0)}, \dot{\varphi}_{(0)}\right)=v_{0}\left(\varphi_{0}, \dot{\varphi}_{0}\right) ;
\end{gathered}
$$

$b^{*}$ the angle elongation limiting conditions, as well as impact conditions

$$
\begin{gathered}
s_{\mathrm{ul}, i}=s_{i}\left(\varphi_{i}\right), \quad s_{\mathrm{ul},(i+1)}=s_{(i+1)}\left(\varphi_{(i+1)}\right), \\
\dot{s}_{\mathrm{odl}, i}\left(\dot{\varphi}_{\mathrm{odl}, i}\right)=-k \dot{s}_{\mathrm{ul}, i}\left(\dot{\varphi}_{\mathrm{ul}, i}\right), \quad \dot{s}_{\mathrm{odl},(i+1)}\left(\dot{\varphi}_{\mathrm{odl},(i+1)}\right)=-k s_{\mathrm{ul},(i+1)}\left(\dot{\varphi}_{\mathrm{ul},(i+1)}\right), \quad i=1,2,3, \ldots, n,
\end{gathered}
$$

where $k$ is impact coefficient, ranging from $k=0$, for the ideal plastic impact, to $k=1$, for the ideal elastic impact; $n$ is total impacts number of a heavy mass particle along the rough parabolic line, up to the equilibrium position, or up to the moment when this particle continues to move without impact with the limiter. The differential equation of the system motion (2.22) cannot be solved explicitly (in the closed form). For their approximate solution we use the software package WOLFRAM Mathematica 7. The results are tested by means of the MATLAB R2008a software package. 


\section{The Analysis of the Vibro-Impact System Motion}

We will divide the motion of a heavy mass particle along the rough parabolic line to the intervals of motion, like this: first interval-from the starting moment of motion to the impact moment with a right side elongation limiter; second interval-from the right side elongation limiter to the impact moment with a left side elongation limiter, and so forth, until the motion direction alternation (motion intervals limited by alternation of friction force direction).

The first motion interval of a heavy mass particle corresponds to the following motion differential equation:

$$
\ddot{\varphi}+(3 \operatorname{tg} \varphi+\mu) \dot{\varphi}^{2}+\frac{g \cos ^{3} \varphi}{p}(\sin \varphi+\mu \cos \varphi)=\frac{F_{0} \cos ^{3} \varphi}{p} \cos \Omega t, \quad \text { for } v>0 .
$$

The impact conditions are

$$
t=t_{\mathrm{ul}_{1+}}, \varphi\left(t_{\mathrm{ul}_{1+}}\right)=\varphi_{1}, \dot{\varphi}\left(t_{\mathrm{ul}_{1}+}\right)=\dot{\varphi}_{\mathrm{ul}_{1}+}
$$

The phase trajectory $\dot{\varphi}_{1}=f(\varphi)$ in the first motion interval (which will be used for the determination of the velocity of heavy mass particle first impact into the right side angular elongation limiter) by using software package Wolfram Mathematica 7 (used also for all the other graphics) is shown in Figure 2.

$$
\begin{gathered}
\text { Parameter values are } \varphi_{1}=\frac{\pi}{4}[\mathrm{rad}], \quad \varphi_{2}=-\frac{\pi}{6}[\mathrm{rad}], \quad \varphi_{0}=0, \quad \dot{\varphi}_{0}=8\left[\frac{\mathrm{rad}}{\mathrm{s}}\right], \\
p=1[\mathrm{~m}], \quad \alpha_{0}=0,05, \quad m=0,2[\mathrm{~kg}], \quad g=9,81\left[\frac{\mathrm{m}}{\mathrm{s}^{2}}\right], \quad F_{0}=0,2[\mathrm{~N}], \quad \Omega=0,3\left[\frac{\mathrm{rad}}{\mathrm{s}}\right] .
\end{gathered}
$$

From the shape of the phase trajectory curve in the first 10 seconds motion interval (Figure 2(b)), we conclude that this trajectory is repeatable during one complete period (from the moment when the heavy mass particle on the rough parabolic line is in initial position to the moment of crossing the same point, in the same direction). This tells us that a heavy mass particle has the same behavior as in the case of free oscillations, despite the influence of external single-frequency force. This influence of external single-frequency force parameters (amplitude and frequency) is negligible. Comparing with the motion without the influence of an external single-frequency force, it can be observed that a heavy mass particle will reach equilibrium position for less motion intervals, that is, reduction of angular velocity value is greater due to elongation limiters impacts.

The external single-frequency force parameters can be chosen in such a way to cause fast reaching of resonant state of a nonimpact vibro-impact motion $\left(F_{0}=0,6-1,0[\mathrm{~N}], \Omega=\right.$ $2,5-3,3[\mathrm{rad} / \mathrm{s}])$. In these cases, research is based on the application of a method that adjusts external single-frequency force parameters in order to obtain a stable periodical vibro-impact regime [26].

The second motion interval (from the first impact into the right side elongation limiter to the second impact into the left side elongation limiter). 


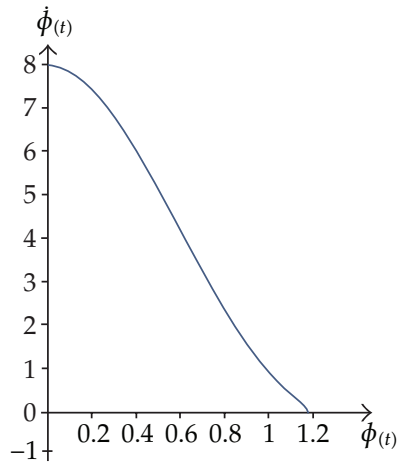

(a)

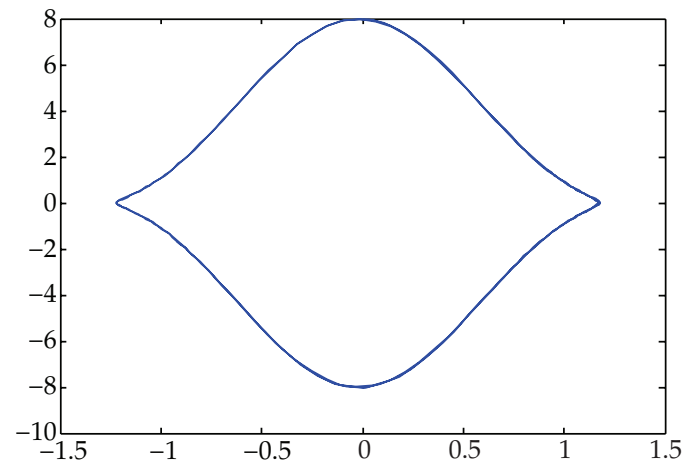

(b)

Figure 2: Phase trajectory curve in the first (nonimpact) motion interval. (a) In time $t=1$ (s), (b) in time $t=10$ (s).

Differential equation of motion has the following form:

$\ddot{\varphi}+(3 \operatorname{tg} \varphi-\mu) \dot{\varphi}^{2}+\frac{g \cos ^{3} \varphi}{p}(\sin \varphi-\mu \cos \varphi)=\frac{F_{0} \cos ^{3} \varphi}{p} \cos \Omega t, \quad$ for $v<0$.

The initial motion conditions are

$$
t=t_{\mathrm{ul}_{1}+}, \quad \varphi\left(t_{\mathrm{ul}_{1}+}\right)=\varphi_{1}, \quad \dot{\varphi}\left(t_{\mathrm{ul}_{1}+}\right)=\dot{\varphi}_{\mathrm{odl}_{1}}=-\dot{\varphi}_{\mathrm{ul}_{1}-} .
$$

The impact condition into the left side elongation limiter is

$$
t=t_{\mathrm{ul}_{2}+}, \quad \varphi\left(t_{\mathrm{ul}_{2}+}\right)=\varphi_{2}, \quad \dot{\varphi}\left(t_{\mathrm{ul}_{2}+}\right)=\dot{\varphi}_{\mathrm{ul}_{2}+} .
$$

The phase trajectory $\dot{\varphi}_{2}=f(\varphi)$ for the other motion interval is shown in the Figure 3.

The motion analysis is conducted to the seventh alternation point, that is, to the moment of motion direction multiple alterations at equilibrium position.

We have noticed that due to influence of an external single-frequency force, there are more phase trajectories around the equilibrium point, in both motion directions of a heavy mass particle along the rough parabolic line. On Figures 4 and 5 there are heavy mass particle phase trajectories after the third alterations point; until the moment of returning to the equilibrium point; Figures 4 and 5 show trajectory for $\dot{\varphi}>0$, and $\dot{\varphi}<0$, respectively.

The graphic visualization of the phase portrait is shown in Figure 6.

The heavy mass particle hits eleven times elongation limiters, five and six impacts into the right side and left side elevation limiters, respectively. Both direction alteration points appear after the eleventh impact into the right side elongation limiter. It means that this vibro-impact system lasts till the eleventh impact into elongation limiters. After the eleventh impact, the analyzed vibro-impact system behaves like a dynamic system, based on forced motion of a heavy mass particle along the rough parabolic line.

For the specified values of parameters, the angular velocity values of a heavy mass particle into elongation limiters and alternation points positions are presented in Table 1. 


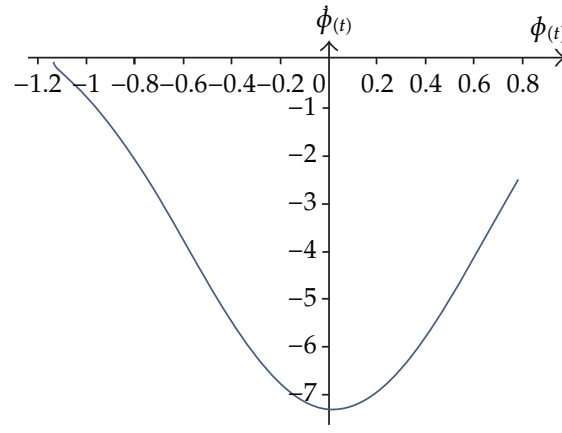

(a)

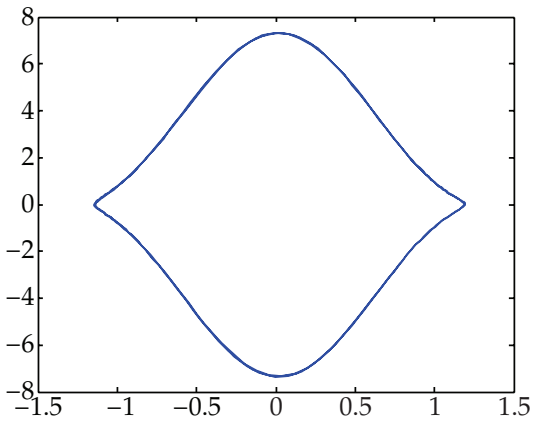

(b)

Figure 3: The phase trajectory curve in the second (non-impact) motion interval. (a) During $t=1$ (s), (b) during $t=10(\mathrm{~s})$.

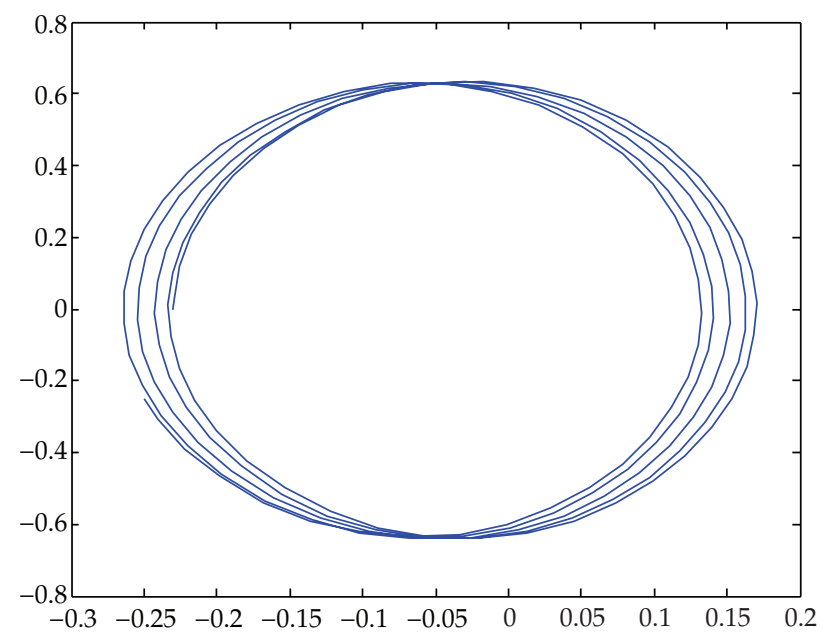

Figure 4: Phase trajectory (after seventh alteration point, $\dot{\varphi}>0), t=10(\mathrm{~s})$.

From Table 1 it can be seen that the angular velocity of impacts into the elongation limiters decreases.

\section{The Energy Analysis of the Vibro-Impact System}

For the energetic analyze of given vibro-impact system, (for each phase portrait branch), an analytical expression that defines dependence of angular velocity value from the generalized coordinate $\varphi$, or time $t$, that is, $\dot{\varphi}=f(\varphi)$ or $\dot{\varphi}=f(t)$, is needed. This analytic dependence represents the phase trajectory equation, which is determined as a first integral of the system motion differential equation (2.22). This equation is a common nonhomogenous and nonlinear differential equation which is being solved by using the software package WOLFRAM Mathematica 7 (solution checked with MATLAB R2008a). It is assumed that the shape of a phase trajectory curve in individual motion intervals is the third order polynomial. In the first motion interval, we have heavy mass particle velocity decrease only. For the rest 


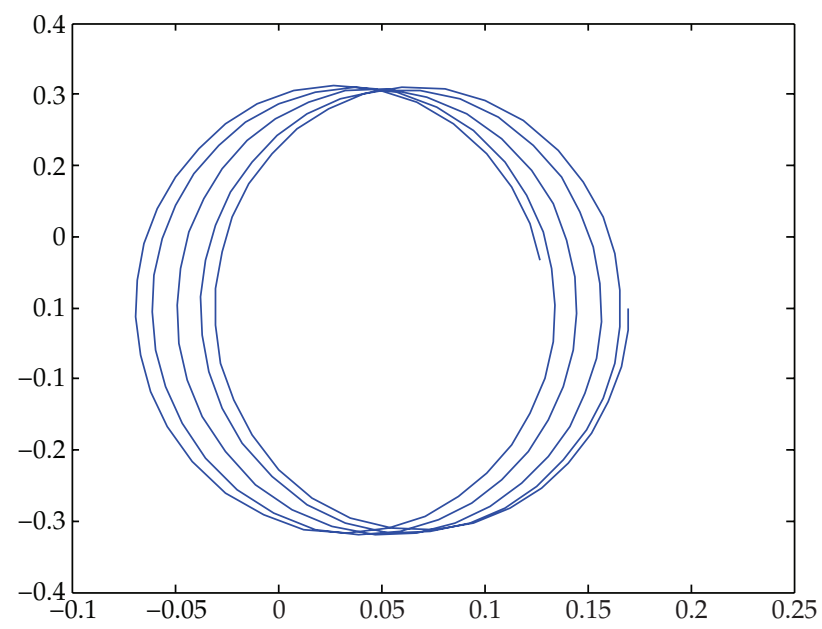

Figure 5: Phase trajectory (after seventh alteration point, $\dot{\varphi}<0), t=10$ (s). Note. The phase trajectory figures are magnified.

Table 1

\begin{tabular}{lccccccccccc}
\hline Number of impacts $(i)$ & $\mathbf{1}$ & $\mathbf{2}$ & $\mathbf{3}$ & 4 & $\mathbf{5}$ & 6 & $\mathbf{7}$ & 8 & $\mathbf{9}$ & 10 & 11 \\
\hline Initial velocities $\varphi_{\mathrm{ul}_{i}}[\mathrm{rad} / \mathrm{s}]$ & 2,49 & 4,41 & 2,02 & 3,65 & 1,60 & 3 & 1,15 & 2,28 & 0,64 & 1,6 & 0,83 \\
Number of alternation points $(j)$ & 1 & 2 & 3 & 4 & 5 & 6 & 7 & & & & \\
$\varphi_{\mathrm{alt}_{j}}[\mathrm{rad}]$ & 0,72 & 0,56 & $-0,43$ & 0,37 & $-0,23$ & 0,17 & $-0,03$ & & & & \\
\hline
\end{tabular}

After the eleventh alteration point, a heavy mass particle is moving within the limits from $-0,3$ to 0,17 . Bold numbers relate to the right-side elongation limiter.

of motion intervals, the phase trajectory curves are divided in two parts. Separation points for those curves are the first points when angular velocity value starts to decrease or increase. These moments are starting data for interpolation, so per one motion interval we have two phase trajectory analytical expressions. If we increase the number of phase trajectory curve sections in one motion interval, during interpolation, it could be obtained the phase trajectory equations with less error (for the phase trajectory curve).

In the Table 2 are shown analytic expressions of phase trajectory equations, obtained by means of interpolation procedure, for the important motion intervals (from the initial position to the seventh point of alternation of a heavy mass particle).

We analyze all motion interval's energy on the basis of kinetic energy $E k$, potential energy $E p$, total mechanic energy $E$ formulas, pressure force $F_{N}$ of a heavy mass particle on the rough parabolic line and power originating of Coulomb sliding friction force $P_{\mu}$, upon determination of analytic expressions $\dot{\varphi}_{i}=f(\varphi)$ :

$$
\begin{gathered}
E k_{i}(\varphi)=\frac{1}{2} m \frac{p^{2}}{\cos ^{6} \varphi} \dot{\varphi}_{i}^{2} ; \quad E p_{i}(\varphi)=\frac{1}{2} \frac{m g p}{\cos ^{2} \varphi} ; \quad E_{i}(\varphi)=E k_{i}(\varphi)+E p_{i}(\varphi)=\frac{1}{2} m \frac{p^{2}}{\cos ^{6} \varphi} \dot{\varphi}_{i}^{2}+\frac{1}{2} \frac{m g p}{\cos ^{2} \varphi} ; \\
F_{N, i}=m g \cos \varphi+\frac{m p}{\cos ^{3} \varphi} \dot{\varphi}_{i}^{2} ; \quad \text { and } P_{\mu, i}=-\mu\left(m g \cos \varphi+\frac{m p}{\cos ^{3} \varphi} \dot{\varphi}_{i}^{2}\right) \frac{p}{\cos ^{3} \varphi} \dot{\varphi}_{i}, \quad i=1,2, \ldots, 18 .
\end{gathered}
$$




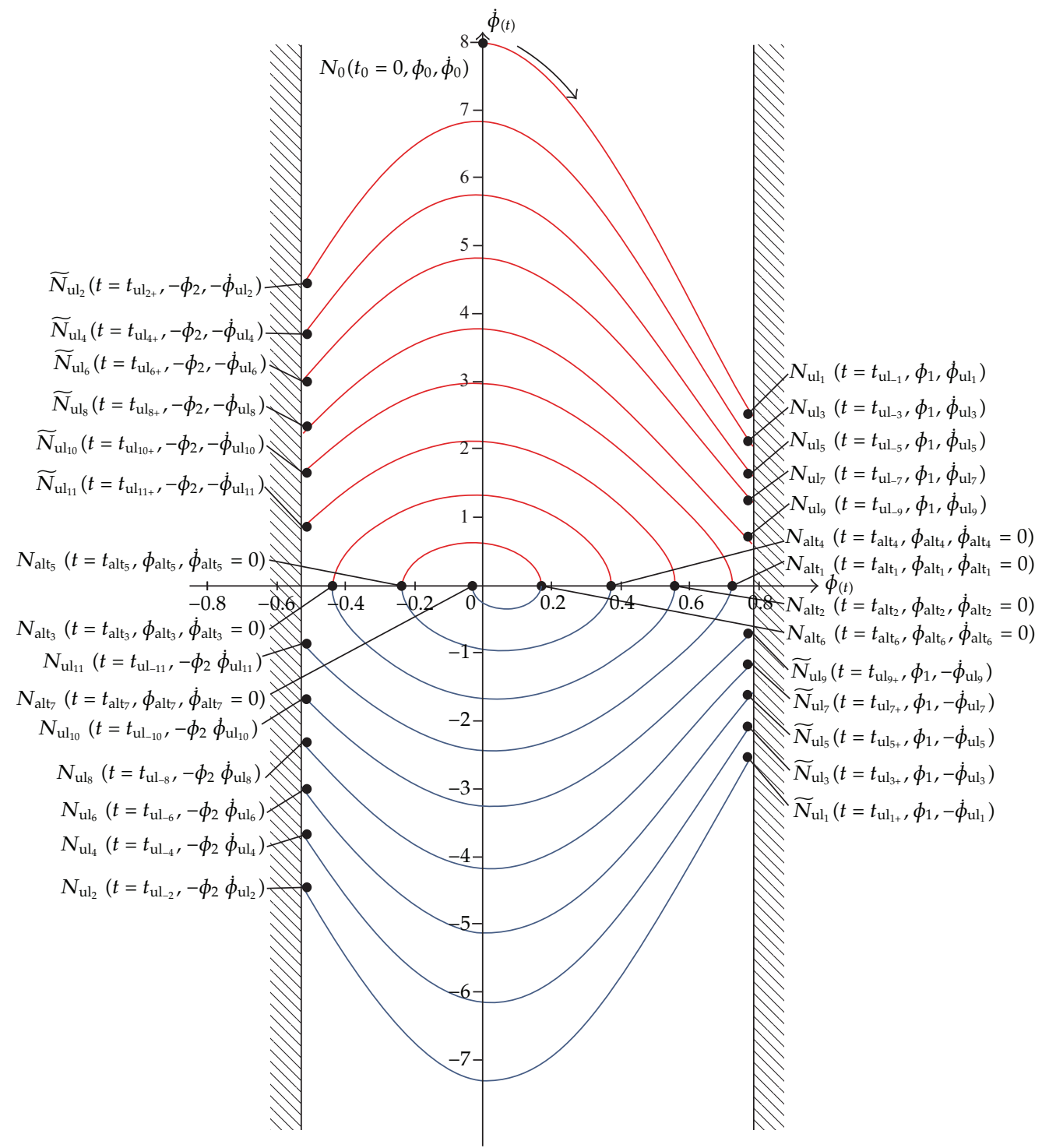

Figure 6: The phase portrait of a heavy mass particle moving along the rough parabolic line, with sliding friction coefficient $\mu=\operatorname{tg} \alpha_{0}$, with two-side limited elongations and under the action of an external singlefrequency force $F(t)=F_{0} \cos \Omega t$, in plane $(\varphi, \dot{\varphi})$.

The energy variations are shown graphically in Figures 7, 8, 9, 10, and 11, presenting the kinetic energy, potential energy, total mechanic energy, heavy mass particle pressure force on the rough parabola for the case of two-side holding bond and Coulomb friction force $\left(\mu=\operatorname{tg} \alpha_{0}\right)$ power (that follows friction force graph) variations, respectively.

From the above graphs which present energetic variations at forced motion of a heavy mass particle along the rough parabolic line, it can be concluded that the influence of an external single-frequency force (for specified parameters $\left(F_{0}, \Omega\right)$ ) is not considerable. It results from the fact that forced motion of a heavy mass particle is out of the resonant region. 
Table 2

\begin{tabular}{lcc}
\hline $\begin{array}{c}\text { Motion } \\
\text { interval }(i)\end{array}$ & \multicolumn{1}{c}{$\dot{\varphi}_{i, 1}$ Phase trajectory equation } & $\dot{\varphi}_{i, 2}$ \\
\hline 1 & $8,7714 \varphi^{3}-15,8705 \varphi^{2}+0,0307 \varphi+7,9882$ & - \\
2 & $-7,9553 \varphi^{3}+15,4219 \varphi^{2}-1,0659 \varphi-7,3009$ & $6,6820 \varphi^{3}+13,4472 \varphi^{2}-0,2817 \varphi-7,3175$ \\
3 & $-5,7113 \varphi^{3}-12,9467 \varphi^{2}-0,6002 \varphi+6,8257$ & $7,6187 \varphi^{3}-13,7678 \varphi^{2}+0,0110 \varphi+6,8208$ \\
4 & $-6,9667 \varphi^{3}+13,4314 \varphi^{2}-0,9975 \varphi-6,1381$ & $5,5926 \varphi^{3}+11,4213 \varphi^{2}-0,3088 \varphi-6,1634$ \\
5 & $-5,1889 \varphi^{3}-11,3992 \varphi^{2}-0,5887 \varphi+5,7231$ & $6,4356 \varphi^{3}-11,6889 \varphi^{2}-0,0639 \varphi+5,7299$ \\
6 & $-5,9559 \varphi^{3}+11,5321 \varphi^{2}-0,8451 \varphi-5,1570$ & $5,0191 \varphi^{3}+10,0426 \varphi^{2}-0,2617 \varphi-5,1719$ \\
7 & $-4,3639 \varphi^{3}-9,7763 \varphi^{2}-0,4786 \varphi+4,8035$ & $5,6025 \varphi^{3}-10,2269 \varphi^{2}-0,0286 \varphi+4,8017$ \\
8 & $-5,0482 \varphi^{3}+9,8710 \varphi^{2}-0,7712 \varphi-4,1828$ & $4,2052 \varphi^{3}+8,5417 \varphi^{2}-0,2774 \varphi-4,1987$ \\
9 & $-3,7709 \varphi^{3}-8,3942 \varphi^{2}-0,4418 \varphi+3,8091$ & $4,1218 \varphi^{3}-8,1458 \varphi^{2}-0,1684 \varphi+3,8155$ \\
10 & $-3,7001 \varphi^{3}+7,9225 \varphi^{2}-0,5620 \varphi-3,2966$ & $3,3867 \varphi^{3}+7,2838 \varphi^{2}-0,2972 \varphi-3,3007$ \\
11 & $-2,9511 \varphi^{3}-7,0920 \varphi^{2}-0,3639 \varphi+2,9311$ & $-1,5676 \varphi^{3}-2,6558 \varphi^{2}-1,1122 \varphi+2,9620$ \\
12 & $3,3223 \varphi^{3}+0,8394 \varphi^{2}+0,9953 \varphi-2,5244$ & $0,8538 \varphi^{3}+5,4223 \varphi^{2}-0,4603 \varphi-2,4430$ \\
13 & $-0,8495 \varphi^{3}-5,4477 \varphi^{2}-0,2065 \varphi+2,0982$ & $-10,0145 \varphi^{3}+2,0863 \varphi^{2}-1,5360 \varphi+2,1247$ \\
14 & $13,3958 \varphi^{3}-5,4903 \varphi^{2}+1,8913 \varphi-1,8138$ & $-18,6681 \varphi^{3}-3,0435 \varphi^{2}-1,2819 \varphi-1,6780$ \\
15 & $30,0382 \varphi^{3}+11,4260 \varphi^{2}+2,4027 \varphi+1,4208$ & $-27,8998 \varphi^{3}+5,0767 \varphi^{2}-1,2206 \varphi+1,3202$ \\
16 & $55,3499 \varphi^{3}-24,0133 \varphi^{2}+4,2842 \varphi-1,1924$ & $-58,0345 \varphi^{3}-2,1824 \varphi^{2}-0,7487 \varphi-0,9341$ \\
17 & $138,8185 \varphi^{3}+34,3472 \varphi^{2}+3,3998 \varphi+0,7216$ & $-143,5033 \varphi^{3}+12,0323 \varphi^{2}-1,0308 \varphi+0,6188$ \\
18 & $557,7284 \varphi^{3}-161,0565 \varphi^{2}+15,9904 \varphi-0,8393$ & $-650,9714 \varphi^{3}+75,6640 \varphi^{2}-3,5338 \varphi-0,2319$ \\
\hline
\end{tabular}

Note. The phase trajectory curve for all motion intervals is divided in two parts; exception is the first interval.

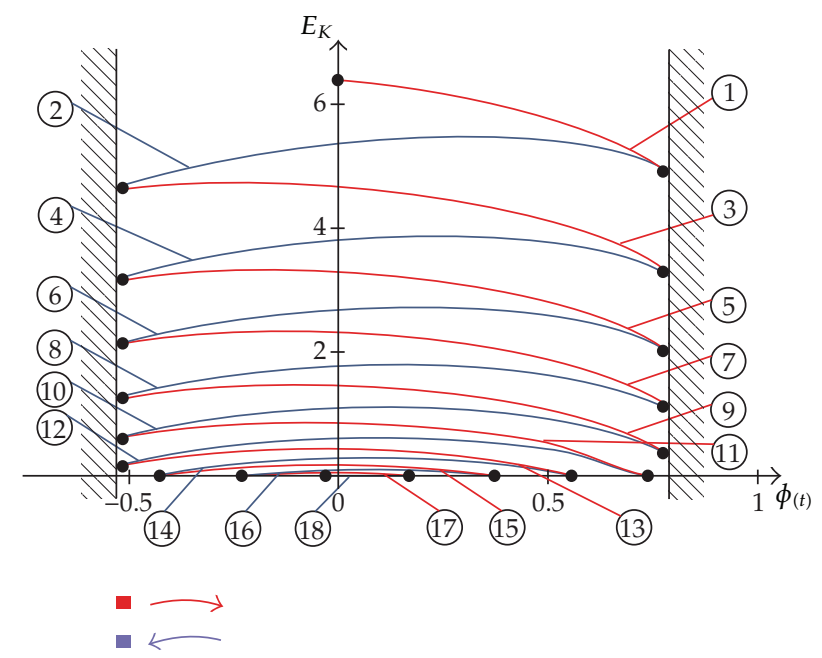

Figure 7: The kinetic energy graph in $(E k, \varphi)$ plane.

\section{Conclusion}

The nonlinearity of given vibro-impact system originates from the discontinuity of a heavy mass particle motion along the rough parabolic line angular velocity. These discontinuities of angular velocity occur in the moment of a heavy mass particle impact into the left- and 


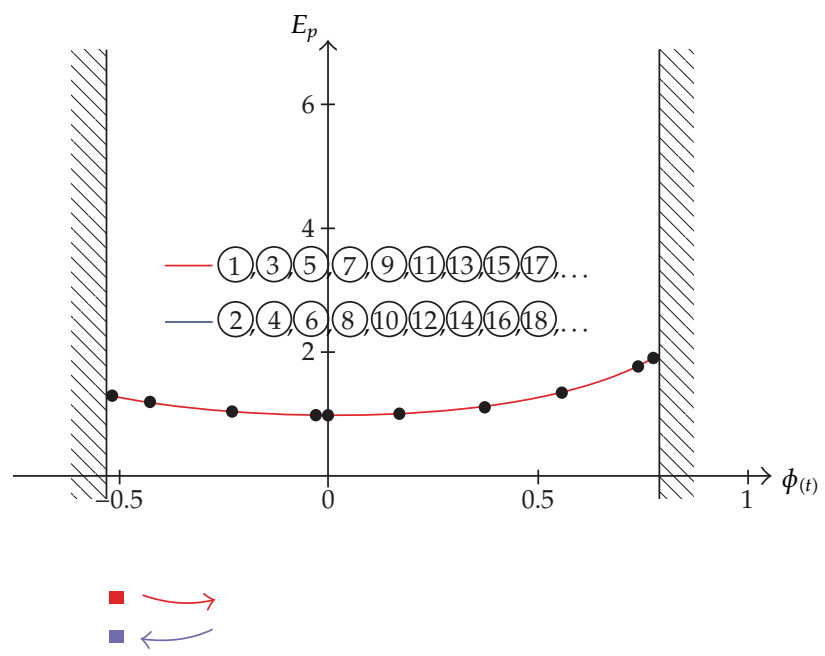

Figure 8: The potential energy graph in $(E p, \varphi)$ plane.

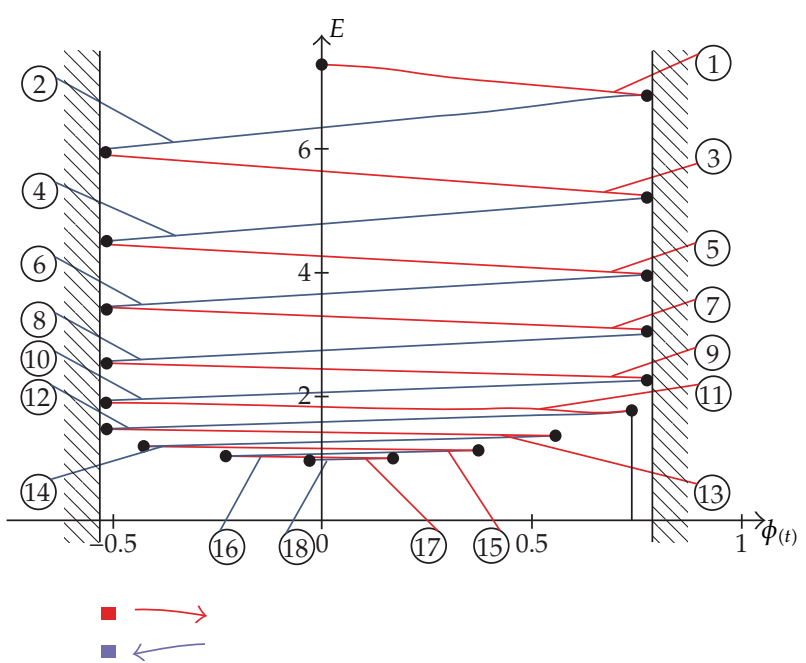

Figure 9: The total energy graph in $(E, \varphi)$ plane.

right-side angular elongation limiters, and in the moment of motion direction alteration of a heavy mass particle, which causes alteration of angular velocity direction and friction force. This nonlinearity is mathematically described, for a heavy mass particle, with a common nonlinear differential equation, specifically with second term that represents a square of angle velocity of generalized coordinate $\dot{\varphi}^{2}$. It corresponds to the case known in the literature as a "turbulent" suppression.

It should be stressed that with given vibro-impact system, due to the influence of Coulomb sliding friction force and alternation of a heavy mass particle angular velocity direction (depending on a heavy mass particle motion direction), there are trigger coupled singularities; that is, there is an equilibrium position bifurcation phenomenon. 


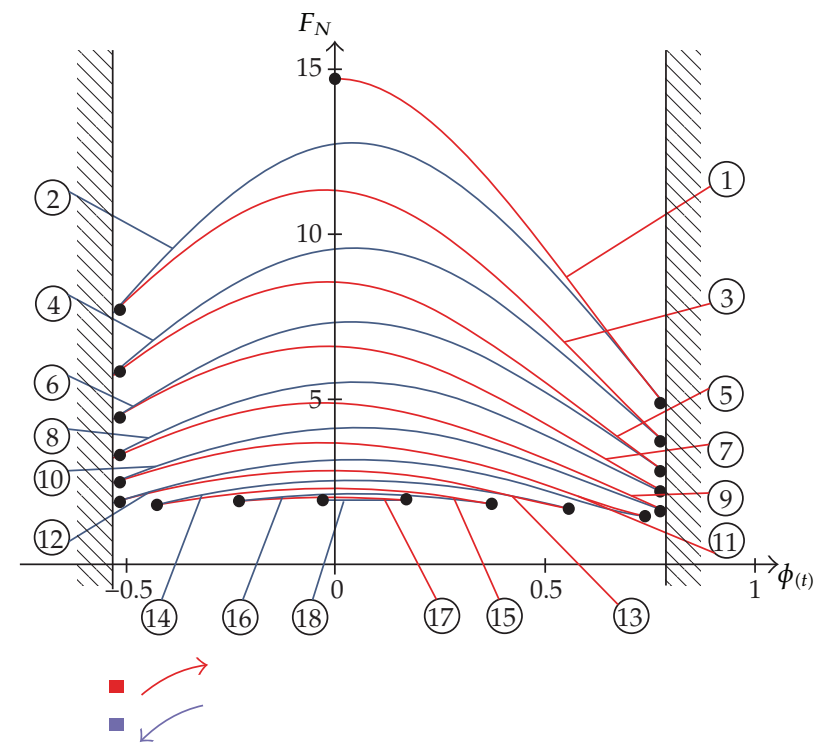

Figure 10: The pressure force graph in $\left(F_{N}, \varphi\right)$ plane.

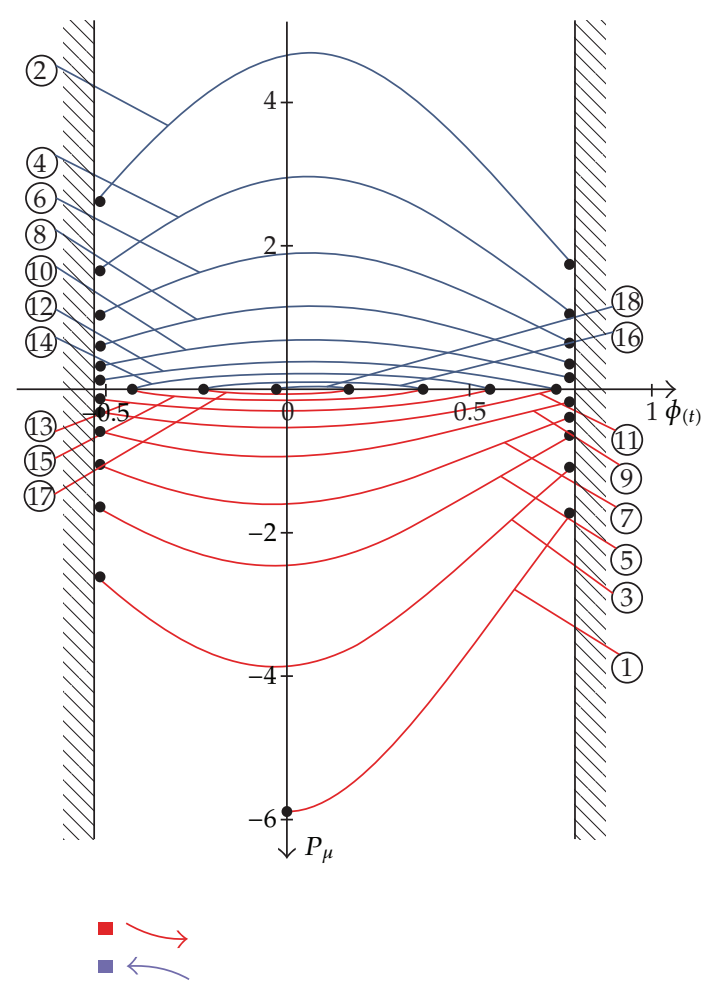

Figure 11: The $P_{\mu}$ power graph in $\left(P_{\mu}, \varphi\right)$ plane. 
The forced motion of a heavy mass particle along the rough parabolic line is divided in corresponding motion intervals and subintervals. To every motion interval and sub-interval corresponds one motion differential equation that belongs to the common nonhomogenous and nonlinear dual differential equation group of the second order. We couldn't solve these differential equations in analytic form. Because of that, we have used the fourth order-variable step Runge-Kutta method that belongs to the numerical methods for a differential equation solving. We have applied the Runge-Kutta method, by using two software packages: MATLAB and Wolfram Mathematica 7 (two equal results obtained). Also, we have joined corresponding initial conditions of motion and impact, to these dual nonlinear and nonhomogenous motion differential equations. The combination of analytic-numerical results for determined kinetic parameters of given vibro-impact system is the basis for motion analyze visualization, that is, for the graphical visualization of phase portrait.

On the basis of known analytical expressions, we have performed the energy analysis of given vibro-impact system, determining analytical expression that defines angle velocity variation dependence from generalized coordinate $\varphi$, that is, $\dot{\varphi}=f(\varphi)$, for each branch of the phase portrait. This analytical dependence that also cannot be determined explicitly is that which we have obtained using the process of phase trajectory curve interpolation. This process of interpolation is done using the MATLAB R2008a software package. It is assumed that the phase trajectory curve shape in specific motion intervals is polynomial of third order.

In the given vibro-impact system, the total mechanic energy dissipates, pressure force onto the rough parabolic line reduces, and friction force power decreases. Also, it should be noted that in the given case, influence of external single-frequency force is not considerable. The cause of that is in the fact that motion of a heavy mass particle is far away from the resonant zone.

\section{Acknowledgments}

Parts of this research were supported by the Ministry of Sciences and Technology of Republic of Serbia through Mathematical Institute SANU Belgrade Grant ON174001.

\section{References}

[1] V. I. Babickii and M. Z. Kolovskii, "Vibrations of linear system with limiters, and excited by random excitation," Mehanika Tverdogo Tela, no. 3, 1967 (Russian).

[2] V. I. Babickii, Theory of Vibro-Impact Systems, Nauka, Moscow, Russia, 1978.

[3] V. I. Babickii and M. Z. Kolovskii, "Investigation of the vibro-impact systems by resonant regimes," Mehanika Tverdogo Tela, no. 4, 1976 (Russian).

[4] J. J. Stoker, Nonlinear Vibrations, Interscience, 1950.

[5] S. Jović, Energijska analiza dinamike vibroudarnih sistema sa krivolinijskim putanjama i neidealnim vezama, doktorska disertacija, Faculty of Technical Sciences, University of Priština, Kosovska Mitrovica, Kosovo, 2010.

[6] F. Peterka, "Laws of impact motion of mechanical systems with one degree of freedom: part Itheoretical analysis of n- multiple (1/n)—impact motions," Acta Technica CSAV, vol. 19, no. 4, pp. 462-473, 1974.

[7] F. Peterka, "Laws of impact motion of mechanical systems with one degree of freedom: part II-resalts of analogue computer modelling of the motion," Acta Technica CSAV, vol. 19, no. 5, pp. 569-580, 1974.

[8] F. Peterka, "Bifurcations and transition phenomena in an impact oscillator," Chaos, Solitons and Fractals, vol. 7, no. 10, pp. 1635-1647, 1996.

[9] S. K. Hedrih, "Nonlinear dynamics of a heavy material particle along circle which rotates and optimal control, chaotic dynamics and control of systems and processes in mechanics," in IUTAM Symposium on Chaotic Dynamics and Control of Systems and Processes in Mechanics, G. Rega and F. Vestroni, Eds., vol. 122 of Solid Mechanics and Its Applications, Editerd by G.M.L.Gladwell, Springer, pp. 37-45, 2005. 
[10] S. K. Hedrih, "Free and forced vibration of the heavy material particle along line with friction: direct and inverse task of the theory of vibrorheology," in Proceedings of the 7th EUROMECH Solid Mechanics Conference, J. Ambrósio et al., Ed., pp. 1-20, Lisbon, Portugal, September 2009.

[11] K. R. Hedrih, "Vibrations of a heavy mass particle moving along a rough line with friction of coulomb type," International Journal of Nonlinear Sciences and Numerical Simulation, vol. 11, no. 3, pp. 203-210, 2010.

[12] S. K. Hedrih, "Discontinuity of kinetic parameter properties in nonlinear dynamics of mechanical systems, Invited Keynote Lecture," in Proceedings of the 9th Brazilian Conference on Dynamics, Control and Their Applications (DINCON '10), pp. 8-40, Serra Negra, Brazil, 2010.

[13] A. H. Nayfeh, "Transfer of energy from high-frequency to Low-frequency modes, Book of Abstracts," in Proceedings of the 2nd International Conference 'Asymptotics in Mechanics', p. 44, St. Petersburg Marine Technical University, St. Petersburg, Russia, October 1996.

[14] A. H. Nayfeh and B. Balachandran, Applied Nonlinear Dynamics, Wiley Interscience, 1995.

[15] M. F. Dimentberg and A. I. Menyailov, "Responce of a single-mass vibroimpact system to white-noise random exitation," ZAMM, vol. 59, pp. 709-716, 1979.

[16] S. Foole and S. Bishop, Bifurcation in Impact Oscillators, UTAM, London, UK, 1998.

[17] G. W. Luo and J. H. Xie, "Hopf bifurcation of a two-degree-of-freedom vibro-impact system," Journal of Sound and Vibration, vol. 213, no. 3, pp. 391-407, 1998.

[18] A. B. Nordmark, "Non-periodic motion caused by grazing incidence in an impact oscillator," Journal of Sound and Vibration, vol. 145, no. 2, pp. 279-297, 1991.

[19] E. Pavlovskaia and M. Wiercigroch, "Periodic solution finder for an impact oscillator with a drift," Journal of Sound and Vibration, vol. 267, no. 4, pp. 893-911, 2003.

[20] E. Pavlovskaia, M. Wiercigroch, K. C. Woo, and A. A. Rodger, "Modelling of ground moling dynamics by an impact oscillator with a frictional slider," Meccanica, vol. 38, no. 1, pp. 85-97, 2003.

[21] K. S. Hedrih, V. Raičević, and S. Jović, "Phase trajectory portrait of the vibro-impact forced dynamics of two heavy mass particles motions along rough circle," Communications in Nonlinear Science and Numerical Simulation, vol. 16, no. 12, pp. 4745-4755, 2011.

[22] K. R. Hedrih, V. Raičević, and S. Jović, "Vibro-impact of a heavy mass particle moving along a rough circle with two impact limiters," International Journal of Nonlinear Sciences and Numerical Simulation, vol. 11, no. 3, pp. 211-224, 2010.

[23] S. K. Hedrih, V. Raičević, and S. Jović, "vibroimpact system dynamics: heavy material particle oscillations along rough circle with two side moving impact limits," in Proceedings of the 2th International Conference on Vibroimpact Systems, pp. 79-86, School of Mechanical Engineering \& Automation Northeastern University, Shenyang, China, January 2010.

[24] K. S. Hedrih, V. Raičević, and S. Jović, "The phase portrait of the vibro-imact dynamics of two mass particle motions along rough circle," in Proceedings of the 3rd International conference on Nonlinear Dynamics, pp. 84-89, Kharkov, Ukraine, Septembar, 2010.

[25] S. Jović and V. Raičević, "Energy analysis of vibro-impact system dynamics based on a heavy mass particle free oscillations along curvilinear rough trajectories," Scientific Technical Review, vol. 60, no. 3-4, pp. 9-21, 2010.

[26] S. Jović and V. Raičević, "Vibro-impact forced oscillations of a heavy mass particle along a rough circle excited by a single-frequency force," Acta Mechanica. In press, http:/ / www.springerlink.com/openurl.asp?genre=article\&id=doi:10.1007/s00707-012-0623-2. 


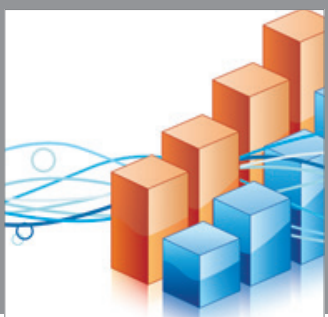

Advances in

Operations Research

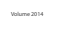

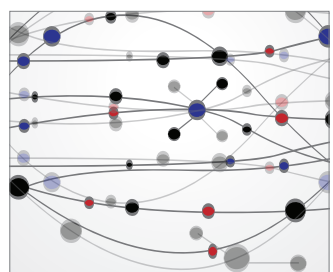

\section{The Scientific} World Journal
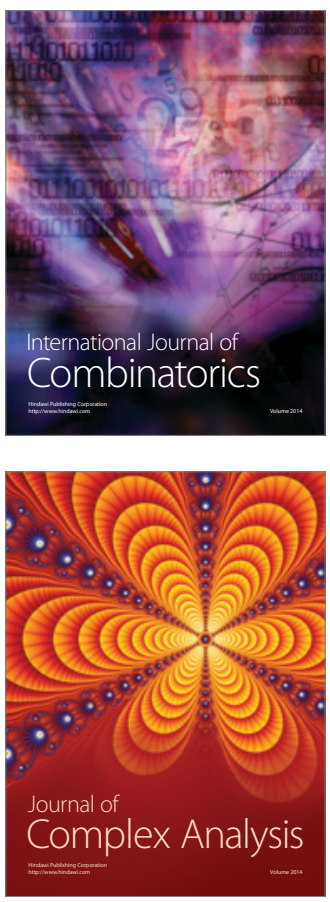

International Journal of

Mathematics and

Mathematical

Sciences
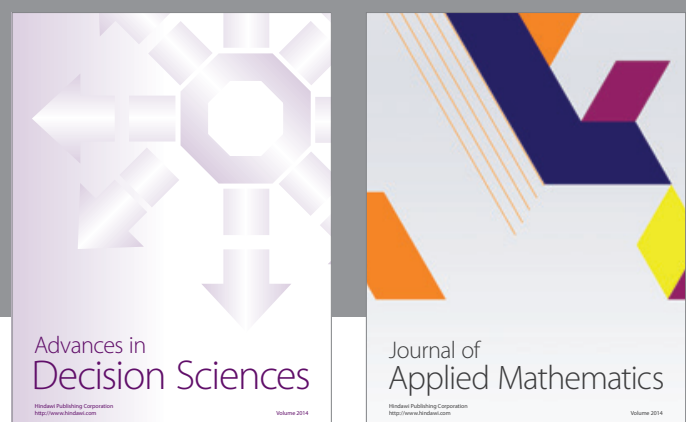

Journal of

Applied Mathematics
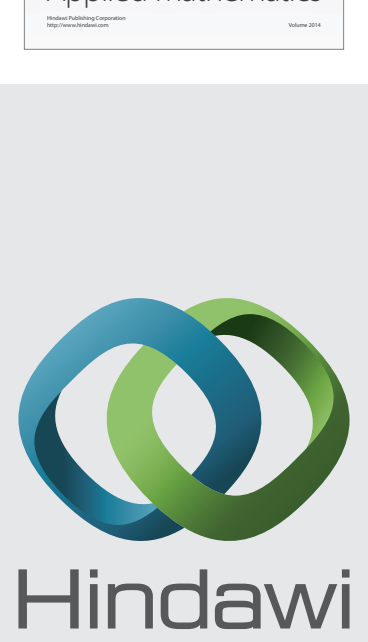

Submit your manuscripts at http://www.hindawi.com
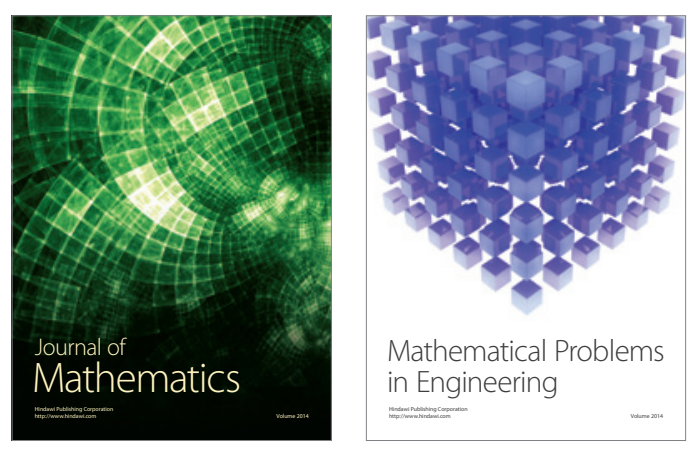

Mathematical Problems in Engineering
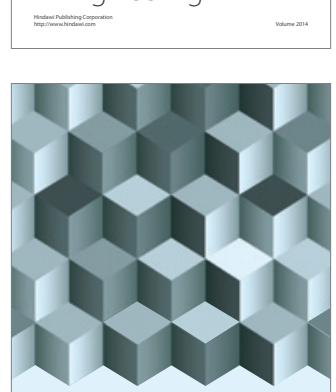

Journal of

Function Spaces
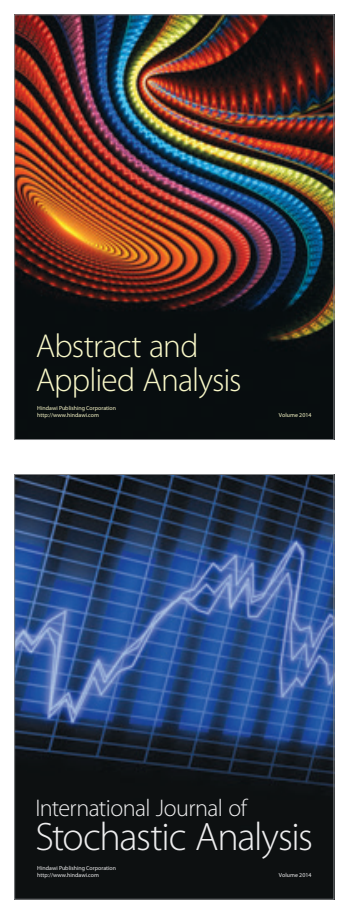

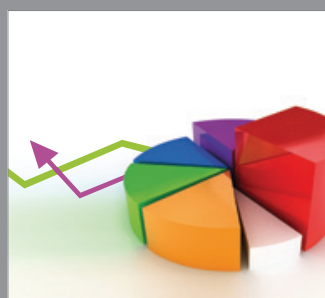

ournal of

Probability and Statistics

Promensencen
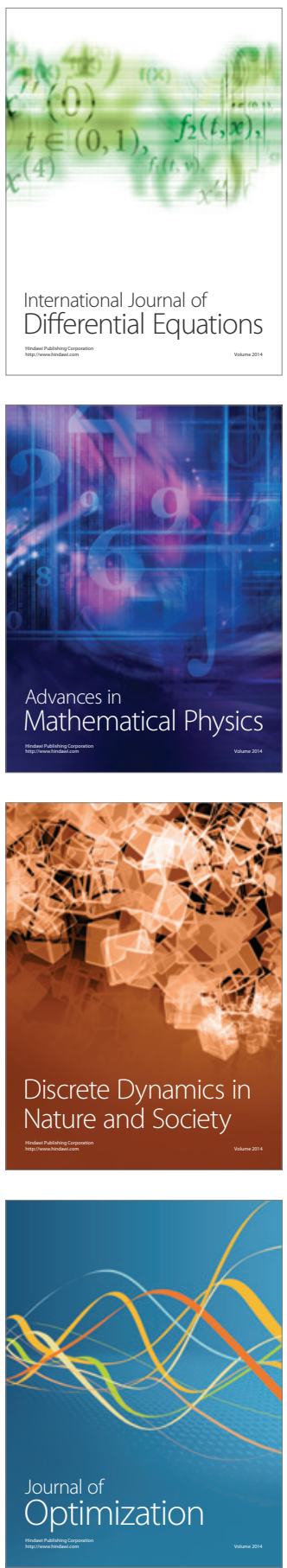